\title{
Autonomy in Technology Design
}

\section{Rafael A. Calvo}

Software Engineering

The University of Sydney

Sydney, NSW 2006 Australia

Rafael.calvo@sydney.edu.au

\section{Daniel Johnson}

CHI Discipline

QId University of Technology

Brisbane, QLD 4001, Australia

dm.johnson@qut.edu.au

\section{Dorian Peters}

Centre for Computer Supported

Learning and Cognition

The University of Sydney

Sydney, NSW 2006 Australia

Dorian.Peters@sydney.edu.au

\section{Yvonne Rogers}

UCL Interaction Centre

University College London

Gower Street,

London WC1E 6BT, UK

y.rogers@ucl.ac.uk

Permission to make digital or hard copies of part or all of this work for personal or classroom use is granted without fee provided that copies are not made or distributed for profit or commercial advantage and that copies bear this notice and the full citation on the first page. Copyrights for third-party components of this work must be honored. For all other uses, contact the Owner/Author.

Copyright is held by the owner/author(s).

CHI 2014, Apr 26 - May 01 2014, Toronto, ON, Canada ACM 978-1-4503-2474-8/14/04.

http://dx.doi.org/10.1145/2559206.2560468

\begin{abstract}
Issues of autonomy impact motivation, the user experience and even psychological wellbeing, yet many questions surrounding design for autonomy remain unanswered. This workshop will explore theory, issues and design strategies related to autonomy drawing on theoretical frameworks available in psychology and looking at autonomy from multiple levels. These include user autonomy within the context of software environments, technologies that increase autonomy in daily life, and how technologies might foster autonomy as a component of psychological development.
\end{abstract}

\section{Author Keywords}

Design methods, design research, gamification, behavior change, self-efficacy, persuasion.

\section{ACM Classification Keywords}

H.5.m [Information Interfaces and Presentation (e.g. $\mathrm{HCI}$ )]: Miscellaneous; H.5.2 [User Interfaces]: Evaluation/Methodology, User-Centered Design; H.5.3[group and Organization Interfaces]:

Evaluation/Methodology;

\section{Introduction}

Autonomy has been defined as, "a state of being independent or self-governing"[14], "a capacity for thinking and acting independently" $[10]$, and more narrowly, as "an internal perceived locus of causality" [12]. It refers at once to the sense of agency required for enjoyable gameplay and to the self-regulation we 


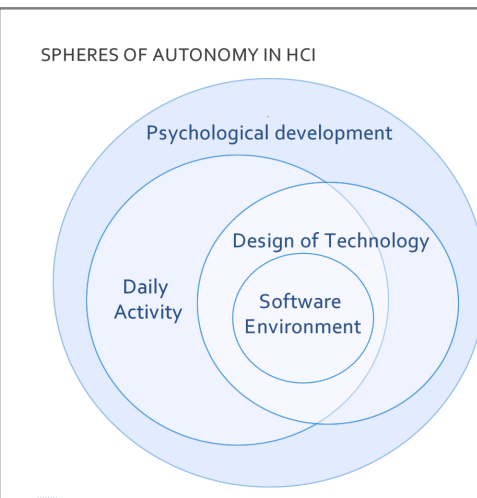

Figure 1. Design for autonomy in $\mathrm{HCI}$ pertains to at least four different spheres: 1 . Within a software environment, 2 . In daily life,

3. In the design of technology 4. For psychological

development and flourishing. often seek to support through behavior change, healthcare and learning technologies. In this workshop we explore autonomy in its broadest sense-as a category of constructs including independence, selfregulation and volition. We favor a broad theme, not only to better allow for open ideation, but also because autonomy-related constructs are so frequently interdependent and inextricable.

Issues of autonomy and how we support it are arguably present in every technology design decision we make $[6,7]$ and yet many questions surrounding design for autonomy in software systems and devices remain unanswered. For example, how often are we designing for compliance at the expense of autonomy? When does reliance on external devices hinder the development of self-regulation? How can technology and its design contribute in a positive way to the support and development of human autonomy? Not only is designing for greater user autonomy relevant from a values sense [6], it is also critical to motivation and wellbeing, a point laid out rigorously in the work of Ryan and Deci $[12,13]$. In this workshop we will explore theory, issues and design strategies related to autonomy in multiple contexts, including software environments, daily life and psychological development.

\section{Spheres of Autonomy in HCI}

Technology has a uniquely pervasive potential to increase or undermine our autonomy at multiple levels. Specifically, when we discuss autonomy in the context of technology, we can view it as pertaining to at least four different spheres (see figure 1). In the narrowest sense, there is design that supports the user in feeling a sense of freedom and control within a software environment. Design for autonomy in this sphere impacts motivation and engagement with a technology, and is very familiar to game designers $[5,8]$. Second, we design for autonomy when we create technologies that give people greater autonomy in daily life (for example assistive technologies, life-tracking, productivity tools, etc.) These are technologies that remove obstacles or enhance capabilities, allowing people to pursue self-determined goals more fluently. Third, there is an emerging notion that users should be empowered to design their own technologies, as suggested by Rogers and Marsden [11] (for example, with kits, or frameworks). Designing for design autonomy empowers users to shape their personal technologies based on their unique and evolving needs and contexts. Finally, and perhaps most significantly, there is the potential to design technologies that foster autonomy as an overarching characteristic of psychological development and flourishing. In other words, how can technologies (eg. educational, health management or behavior change tools) be designed to help users develop autonomy (and related concepts like self-efficacy, independence and self-regulation) over time as a trait, characteristic or capacity?

\section{From self-tracking to self-reliance}

By way of example, consider systems designed for people with chronic disease. Young people with chronic illness are required to transition from paediatric to adult care, which includes becoming self-reliant managers of their health. In relation to the first sphere, any technologies developed to support this process would support user autonomy within the context of use (eg. freedom to choose features and set parameters). In addition, text might be written to avoid authoritarian tone (eg. "considered a walk around the block? " v. "Go take a walk.") Moving into the second sphere, tools 
might increase patient autonomy in their daily lives in ways that support their transition, for example by providing access to information, or opportunities to connect with peers. In relation to the third sphere, we might imagine tools that allow patients to develop or highly customise their own transition support technologies. And finally, to successfully support a genuine transition from parent-dependent youth to selfreliant adult, technology to foster autonomy as a psychological strength would develop a patient's capacity to self-manage their care (for example by providing adaptive and gradually fading support in the way teachers and therapists do). This would support patients in lasting autonomy, throughout their lives even as their needs, their technologies, and their care evolve.

This example combines the existing and the hypothetical. But how do we identify strategies for "autonomy-supportive" design, including both those that are already employed in current technologies, and those we have yet to envision? Furthermore, how do we obtain empirical evidence of which designs are more effective? In this workshop we will seek to

collaboratively investigate this space, leveraging the work and experience brought by participants, and drawing on various theoretical frameworks related to autonomy and self-efficacy.

\section{Theoretical frameworks}

A number of psychological theories can be used in $\mathrm{HCI}$ as theoretical frameworks for understanding the impact of design strategies on autonomy and related concepts. Self-Determination Theory (SDT) addresses socialcontextual factors that facilitate or undermine selfmotivation and healthy psychological development [12] and specifies autonomy, competence and relatedness as the three pillars of motivation and wellbeing. As part of SDT, Cognitive Evaluation Theory (CET) specifies factors that explain variability in intrinsic motivation [13] focusing specifically on the fundamental needs of competence and autonomy. Competence refers to a need for optimal challenge (a good match between skills and task) and feelings of effectance. Autonomy refers to willingness or a sense of volition around an activity. Autonomy is maximised when a task is done for interest or personal value and can be facilitated by features of the environment including the provision of choice and non-controlling instructions.

We can also draw on Bandura's [1,2] theory of selfefficacy, which suggests that people will expend and sustain effort in the face of challenges as a function of their sense of personal efficacy. A person's sense of self-efficacy is derived from their beliefs about their performance accomplishments, vicarious experience, verbal/social persuasion and psychological state. We anticipate workshop participants will bring further theoretical frameworks to the table and a goal of the workshop will be bringing these various approaches and perspectives together for future research.

\section{Workshop goals and themes}

Together we will look at current and hypothetical examples of impact on autonomy. For example:

- Games - What can we learn from how game designers design for autonomy? How might we design beyond in-game autonomy?

- Internet of things - How can advances in ubiquitous computing support or undermine autonomy (eg. monitoring, tracking) 
- Assistive technologies and HCI4D - How can we move from "augmenting frailty" to a "rhetoric of empowerment"?

- Personal Informatics [9] - What design decisions impact self-regulation?

- Persuasive Technologies - How might theories of autonomy interact with Fogg's model of behaviour change? [4]

- Learning Technologies - What can we learn from education research on the development of independence and self-efficacy?

\section{References}

[1] Bandura, Albert. "Self-efficacy: toward a unifying theory of behavioral change."Psychological review 84.2 (1977): 191.

[2] Bandura, Albert. Self-efficacy. John Wiley \& Sons, Inc., 1994.

[3] Calvo, R., \& Peters, D. (2014). Positive Computing (p. to appear). Cambridge, MA: MIT Press.

[4] Fogg, Brian J. "Persuasive technology: using computers to change what we think and do." Ubiquity 2002.December (2002): 5.

[5] Ford, Matthew, Peta Wyeth, and Daniel Johnson. "Self-determination theory as applied to the design of a software learning system using whole-body

controls." Proceedings of the 24th Australian ComputerHuman Interaction Conference. ACM, 2012.

[6] Friedman, Batya. "Value-sensitive design." Interactions 3.6 (1996): 16-23.

[7] Friedman, Batya, Peter H. Kahn Jr, and Alan Borning. "Value sensitive design and information systems." Human-computer interaction in management information systems: Foundations 5 (2006): 348-372.
A better understanding of how to support and develop autonomy would clearly benefit the work of our community, resonating through many subfields from $\mathrm{HCI}$ in health, sustainability, development and wellbeing to personal informatics and ubiquitous computing [3]. This workshop aims to contribute a more organized way of thinking about issues surrounding autonomy, self-efficacy and self-regulation by clarifying the issues, terminology and available theoretical frameworks available to inform autonomysupport in technology design.

[8] Johnson, D., \& Gardner, J. (2010). Personality, motivation and video games. In Proceedings of the 22nd Conference of the Computer-Human Interaction Special Interest Group of Australia on ComputerHuman Interaction (pp. 276-279). ACM.

[9] Li, Ian, Anind Dey, and Jodi Forlizzi. "A stage-based model of personal informatics systems." Proceedings of the SIGCHI Conference on Human Factors in Computing Systems. ACM, 2010.

[10] Littlewood, W. (1996). Autonomy: An Anatomy and a Framework. System, 24(4).

[11] Rogers, Y., \& Marsden, G. (2013). Does he take sugar? interactions, 20(4), 48.

[12] Ryan, R., \& Deci, E. (2000). Intrinsic and Extrinsic Motivations: Classic Definitions and New Directions. Contemporary educational psychology, 25(1), 54-67.

[13] Ryan, R. M., Rigby, C. S., \& Przybylski, A. (2006). The Motivational Pull of Video Games: A Self-

Determination Theory Approach. Motivation and Emotion, 30(4), 344-360.

[14] Spear, H. J., \& Kulbok, P. (2001). Autonomy and adolescence: a concept analysis. Public health nursing (Boston, Mass.), 21(2), 144-52. 